\title{
Application of TITAN for Simulation of Particle Streaming in a Duct
}

\author{
Katherine Royston ${ }^{1, \mathrm{a}}$, Alireza Haghighat ${ }^{1}$, and $\mathrm{Ce} \mathrm{Yi}^{2}$ \\ ${ }^{1}$ Virginia Tech, 900 N. Glebe Rd., Arlington, Virginia, USA \\ ${ }^{2}$ Georgia Tech, 770 State St., Atlanta, Georgia, USA
}

\begin{abstract}
The TITAN hybrid deterministic transport code is applied to the simulation of particle streaming in a nuclear power plant duct. A simple model is used consisting of a concrete duct emerging from the pressure vessel with an isotropic surface source with a U-235 fission spectrum located at the pressure vessel end. Multiple methods of simulating the duct using the TITAN code are considered to demonstrate the flexibility of the code and the advantages of TITAN's algorithms. These methods include a discrete ordinates $\left(S_{N}\right)$ calculation, a characteristics method calculation, and the use of a fictitious quadrature set with simplified ray-tracing. The TITAN code's results are compared with MCNP5 solutions. While all TITAN solutions are obtained in a shorter computation time than the MCNP5 solution, the TITAN solution with the fictitious quadrature set shows the largest speedup.
\end{abstract}

\section{Introduction}

The simulation of particle streaming through a duct penetrating the pressure vessel in a BWR presents difficulties for both Monte Carlo and deterministic methods. In a Monte Carlo simulation, the computation time necessary to achieve reasonable statistical uncertainties can be large because few particles will make it through the duct to reach the detector. In a deterministic simulation, memory can be a limitation, because the angular discretization may require a large number of directions to accurately represent a narrow duct. The TITAN hybrid deterministic transport code [1] has methods that allow it to efficiently represent regions with low scattering media, e.g., air in a duct. These methods are applied to a model of a duct leaving a BWR pressure vessel.

In Sect. 2, the TITAN code's methods are introduced: i) a hybrid discrete ordinates and characteristics method and ii) a fictitious quadrature with simplified ray-tracing method. Section 3 describes the model details and reference Monte Carlo solution and Sect. 4 presents results. Finally, Sect. 5 gives conclusions.

\section{The TITAN code}

The TITAN code is a parallel deterministic radiation transport code that solves the linear Boltzmann equation. The code was initially developed with hybrid methods for medical physics applications, which

\footnotetext{
${ }^{\text {a }}$ Corresponding author: royston@vt.edu
}

This is an Open Access article distributed under the terms of the Creative Commons Attribution License 2.0, which permits unrestricted use, distribution, and reproduction in any medium, provided the original work is properly cited. 


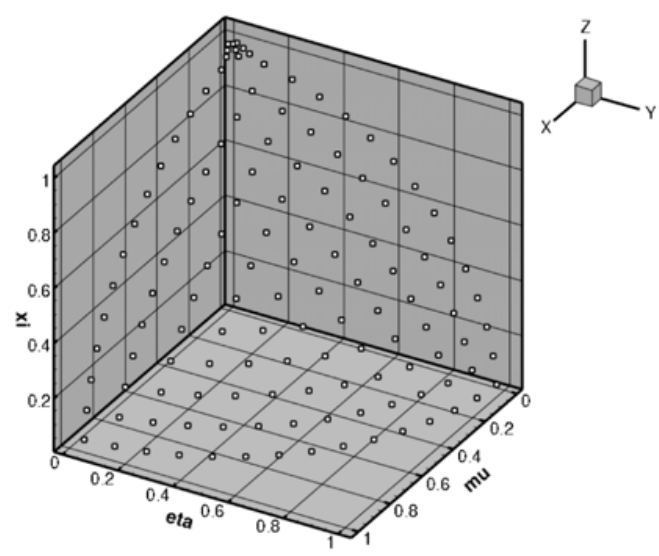

Figure 1. $\mathrm{S}_{30}$ Legendre-Chebyshev quadrature set with ordinate splitting of the direction closest to the z-axis (128 directions per octant).

commonly contain large air regions. These hybrid methods can also be applied to applications with low scattering media, such as a duct filled with air.

\subsection{Hybrid Discrete Ordinates and Characteristics Method}

The hybrid discrete ordinates $\left(\mathrm{S}_{\mathrm{N}}\right)$ and characteristics method $(\mathrm{CM})$ is based on the TITAN code's blockoriented framework. The block spatial meshing divides the problem into coarse meshes and each coarse mesh can use either an $S_{N}$ solver or a characteristics solver. Coarse meshes using the $S_{N}$ solver are filled with uniform fine meshes and those using the characteristics solver are filled with characteristic rays. All coarse meshes use the same energy and angle discretization methods; however, different quadrature sets can be specified in different coarse meshes.

If a particular direction is important in a model, generally one must increase the angular discretization over the entire angular space by increasing the quadrature order. This will significantly increase the memory required and slow down the calculation. The TITAN code includes the ordinate splitting technique [2] that can be used to refine specific directions without increasing the base quadrature order. Figure 1 gives an example of an $\mathrm{S}_{30}$ Legendre-Chebyshev quadrature set with ordinate splitting of the direction closest to the z-axis.

\subsection{Fictitious Quadrature Set}

The fictitious quadrature set with simplified ray-tracing [3] was initially developed for simulating medical projection images at different angles around a phantom with a source. The formulation for generating projection images can be summarized in four steps:

i. Discrete ordinates transport calculation in the phantom with a regular quadrature set;

ii. Generation of a fictitious quadrature set with circular ordinate splitting (COS) for a projection angle;

iii. A single extra transport sweep through the phantom using converged flux moments from Step $i$ to evaluate the scattering source; 


\section{$15^{\text {th }}$ ISRD}

iv. Simulation of a projection image with the fictitious quadrature set using a simplified ray-tracing formulation outside of the phantom.

Step $i$ only needs to be completed once and is generally the most time consuming calculation. Steps $i i-i v$ are then repeated for each projection angle.

The fictitious quadrature set was developed for when the angular flux is needed in directions that are not necessarily included in the problem quadrature set. The directions of interest are specified in the fictitious quadrature set, which is used with an extra transport sweep after the source iteration process is converged (Step iii). The scattering source along the fictitious quadrature set directions is then determined using the converged flux moments, as in Eq. (1).

$$
\begin{aligned}
S_{\text {scattering }}^{(e . s .)} & \sum_{g^{\prime}=1}^{G} \sum_{l=0}^{L}(2 l+1) \sigma_{s, g^{\prime} \rightarrow g, l}\left\{P_{l}\left(\mu_{n}^{(f i c)}\right) \cdot \phi_{g^{\prime}, l}^{(c o n)}\right. \\
& \left.+2 \sum_{k=1}^{l} \frac{(l-k) !}{(l+k) !} P_{l}^{k}\left(\mu_{n}^{(f i c)}\right) \cdot\left[\phi_{C, g^{\prime}, l}^{k,(c o n)} \cos \left(k \varphi_{n}^{(f i c)}\right)+\phi_{S, g^{\prime}, l}^{k,(c o n)} \sin \left(k \varphi_{n}^{(f i c)}\right)\right]\right\} .
\end{aligned}
$$

In Eq. (1), e.s. stands for extra sweep, fic for fictitious, and con for converged. The $\phi_{g^{\prime}, l}^{(c o n)}, \phi_{g^{\prime}, l}^{(c o n)}$, and $\phi_{g^{\prime}, l}^{(c o n)}$ terms are the converged $l^{\text {th }}$ order regular, cosine, and sine flux moments, respectively, and $\left(\mu_{n}^{(f i c)}, \varphi_{n}^{(f i c)}\right)$ specifies a direction in the fictitious quadrature set.

To represent collimation, the COS technique in TITAN splits the detector normal into several directions on a circle about the normal to create the fictitious quadrature set. The angular flux leaving the phantom along the directions is then averaged to approximate collimator blur. The weighted circular ordinate splitting (WCOS) technique [4] was created to improve the accuracy of the COS technique for large collimator acceptance angles [5]. In the WCOS technique, the fictitious quadrature set directions are used to calculate a weighted average of the overlap of the detector surface projected along the direction to the collimator front.

\section{Problem Description}

A simple concrete duct leaving a pressure vessel is modelled to simulate neutron streaming from a nuclear reactor duct. The model dimensions are $100 \mathrm{~cm}$ by $100 \mathrm{~cm}$ by $762 \mathrm{~cm}$ and the air duct has a cross-section of $40 \mathrm{~cm}$ by $40 \mathrm{~cm}$ and is exiting a $10 \mathrm{~cm}$ thick steel pressure vessel, as depicted in Fig. 2 . An isotropic surface source with a U-235 fission spectrum is located at the pressure vessel end of the duct as a simple surrogate for a reactor core. The average flux over the exit at the far end of the duct is calculated. The BUGLE-96 47-group neutron cross-section library is used.

\subsection{Deterministic Model}

A variety of deterministic methods are used to simulate the duct problem. These methods are summarized as:

Case 1. TITAN $\mathrm{S}_{\mathrm{N}}$ only model;

Case 2. TITAN $\mathrm{S}_{\mathrm{N}}$ model with characteristics method in air;

Case 3. TITAN $\mathrm{S}_{\mathrm{N}}$ with fictitious quadrature and simplified ray-tracing model.

In Case 1, the $S_{N}$ solver is used throughout the model. The air region inside the duct uses the characteristics solver in Case 2, while the rest of the model uses the $S_{N}$ solver. Case 3 only models the pressure vessel region of the duct (the first $10 \mathrm{~cm}$ ) and uses the $S_{N}$ solver. As described in 

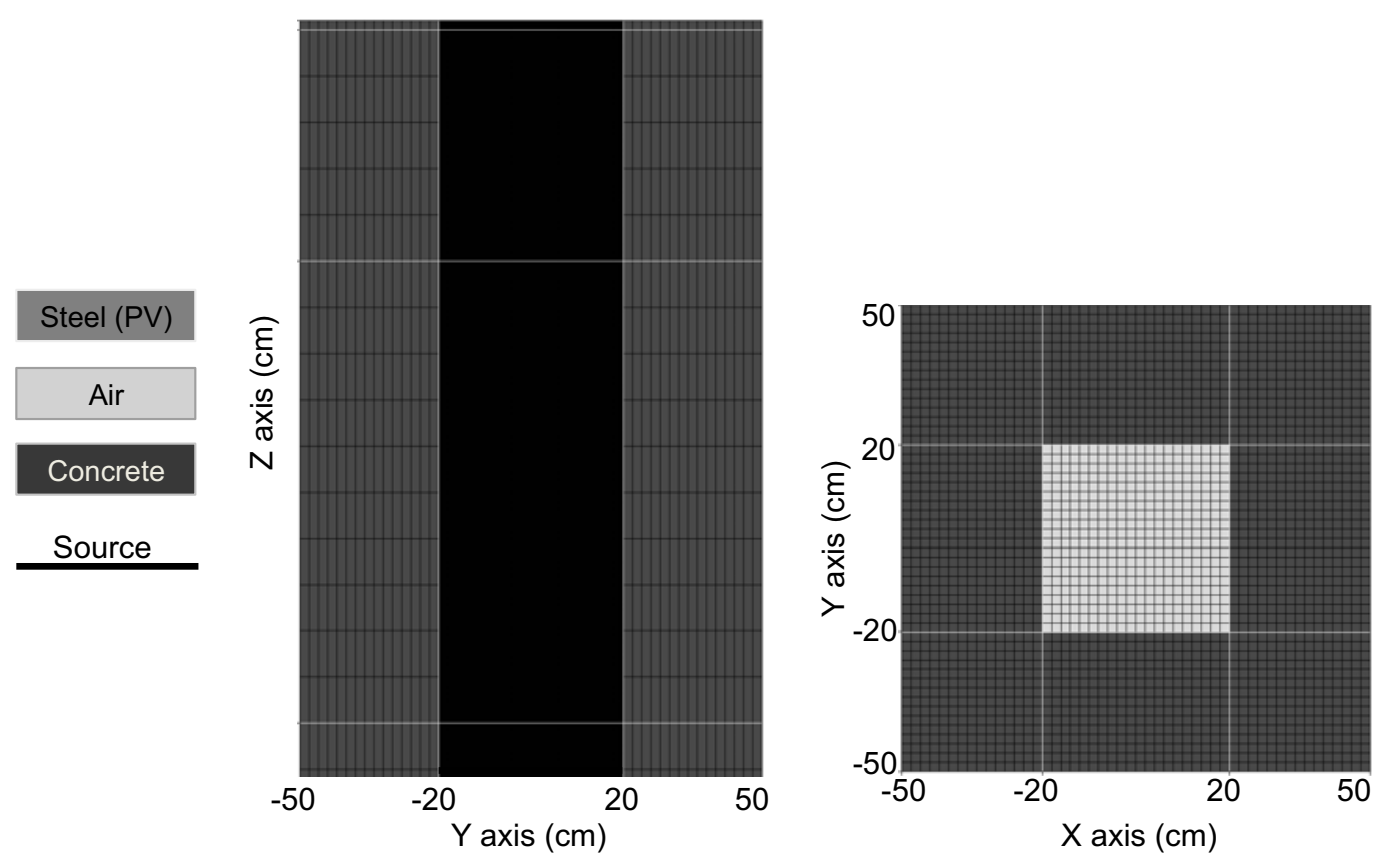

Figure 2. Duct geometry and base meshing with surface source located at the duct entrance (note that duct length along the $\mathrm{z}$-axis is cropped here).

Sect. 2.2, the fictitious quadrature set is then used to determine the angular flux reaching the end of the duct. The fictitious quadrature set does not conserve flux moments [3] so results are scaled to match the peak flux value for comparison. All TITAN cases used a flux tolerance of $1 \times 10^{-3}$ to determine convergence.

The base meshing depicted in Fig. 2 has 195,000 fine meshes in Case 1. Fine meshes are $2 \mathrm{~cm}$ by $2 \mathrm{~cm}$ by $5 \mathrm{~cm}$ in the pressure vessel and $2 \mathrm{~cm}$ by $2 \mathrm{~cm}$ by $10 \mathrm{~cm}$ elsewhere. In Case 2, the coarse meshes using the characteristics method have one fine mesh so the memory required is reduced. Case 3 only models the pressure vessel region so only 7500 fine meshes are needed.

\subsection{Reference Monte Carlo Model}

The MCNP5 Monte Carlo code [6] was used to produce reference solutions. A mesh tally at the far end of the duct is used to determine the flux and 16 billion particle histories were simulated. All energy groups had $1 \sigma$ uncertainty less than $3.3 \%$, except for groups 1 and 2 which are not resolved. The XSMCNP utility code [7] was used to convert the deterministic cross sections to MCNP's multigroup format. It is worth noting, that while the cross sections are generated from those used in TITAN to minimize differences between the two codes, the deterministic scattering moments cannot be directly used by MCNP so some differences in cross sections will remain.

\section{Results}

Results are first presented looking at the behaviour of the TITAN simulation for different deterministic parameters. The methods are compared with each other as well as a reference MCNP solution. A comparison of computation times is also given. 


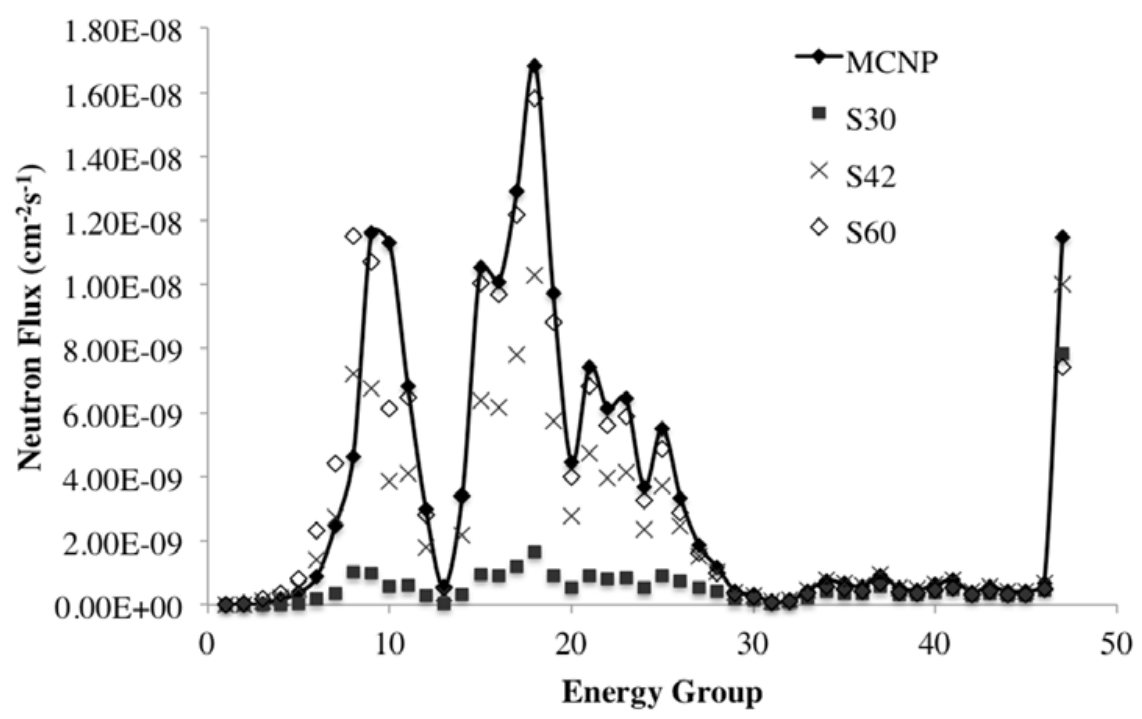

Figure 3. Flux spectra averaged over the end of the duct for TITAN $S_{N}$ calculations with $S_{30}$ (960 directions), $\mathrm{S}_{42}$ (1848 directions), and $\mathrm{S}_{60}$ (3720 directions) quadrature sets and reference MCNP solution.

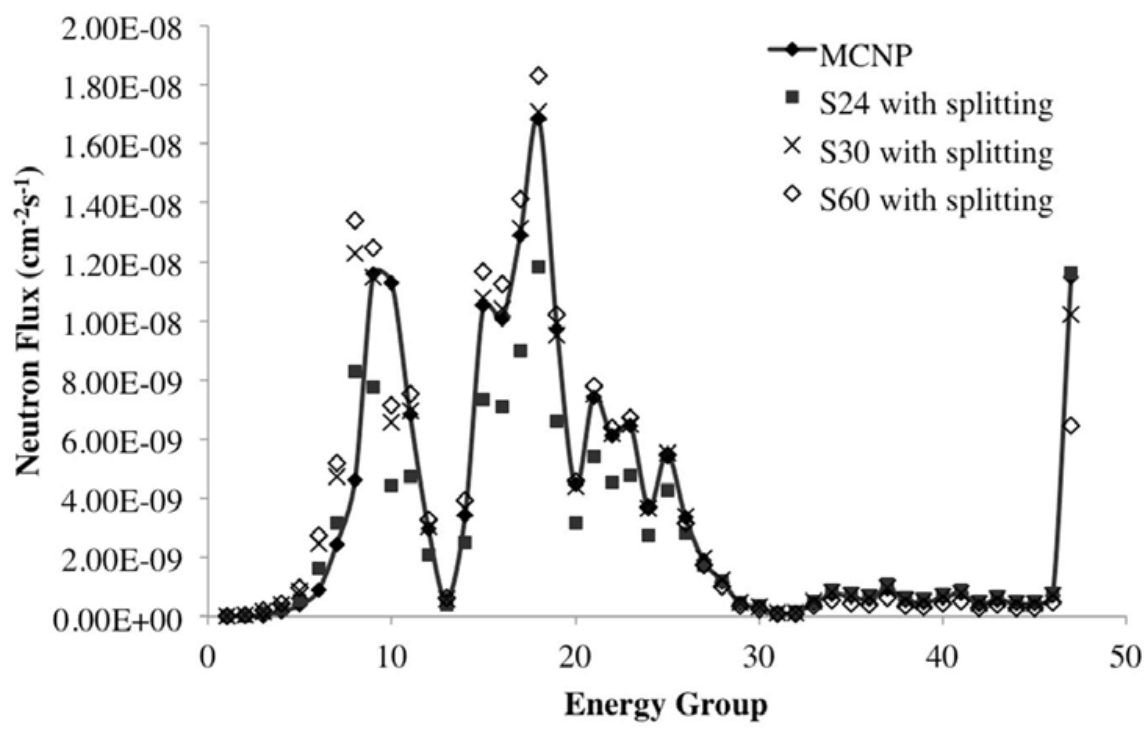

Figure 4. Flux spectra averaged over the end of the duct for TITAN $S_{N}$ calculations with $S_{24}$ with splitting (688 directions), $\mathrm{S}_{30}$ with splitting (1024 directions), and $\mathrm{S}_{60}$ with splitting (3784 directions) quadrature sets and reference MCNP solution.

\section{1 $\mathrm{S}_{\mathrm{N}}$ Algorithm Studies}

Studies were completed using only the $S_{N}$ solver to choose the appropriate deterministic parameters. The $\mathrm{P}_{1}$ anisotropic scattering order is used after the $\mathrm{P}_{3}$ solutions were shown to only change by a few percent. This is expected because scatter will be a minor contribution to the flux exiting the duct. The appropriate quadrature order and meshing were then determined.

The impact of quadrature order is examined in Figs. 3 and 4 for base meshing with only the $S_{N}$ solver without and with ordinate splitting, respectively. As discussed in Sect. 2.1, the ordinate splitting 
Table 1. TITAN number of directions, total flux difference relative to MCNP $(1 \sigma=0.05 \%)$, and computation time on 16 processors for $\mathrm{S}_{\mathrm{N}}$ calculations with different quadrature sets.

\begin{tabular}{lccc}
\hline Quadrature Order & Number of Directions & Relative Difference & Computation Time (s) \\
\hline S30 & 960 & $-82.0 \%$ & 3359 \\
S30 with splitting & 1024 & $5.21 \%$ & 3479 \\
S60 & 3720 & $-5.73 \%$ & 12406 \\
S60 with splitting & 3784 & $6.80 \%$ & 12575 \\
\hline
\end{tabular}

technique can be used to reduce the number of directions needed. The cases in Fig. 4 have the direction that is closest to the z-axis split into 9 directions in each octant, as shown in Fig. 1.

In Fig. 3, the $S_{30}$ quadrature set is clearly inadequate, but in Fig. 4 the $S_{30}$ quadrature with splitting gives a good match to the reference MCNP data. In order to achieve a converged solution without using ordinate splitting, an $\mathrm{S}_{60}$ quadrature set was needed. Table 1 summarizes the number of directions, total flux difference relative to MCNP $(1 \sigma=0.05 \%)$, and computation time on 16 processors for the $S_{30}$ and $\mathrm{S}_{60}$ quadrature sets with and without splitting. Ordinate splitting enables an accurate solution to be obtained using 1024 directions instead of 3720 directions. The $S_{30}$ quadrature with ordinate splitting is used in all following calculations.

The sensitivity to mesh size was examined next. The number of fine meshes along the z-axis was reduced to create a model with $2 \mathrm{~cm}$ by $2 \mathrm{~cm}$ by $25 \mathrm{~cm}$ fine mesh dimensions for a total of 82,500 fine meshes. The results of this reduced-z model differ by less than $6 \%$ across all energy groups from the base meshing solution and the computation time is halved. Another calculation was run with the number of meshes in $x$ and $y$ doubled while using the reduced- $z$ meshing for a fine mesh dimension of $1 \mathrm{~cm}$ by $1 \mathrm{~cm}$ by $25 \mathrm{~cm}$ and 330,000 fine meshes total. This refined-xy flux spectrum was within $5 \%$ of the base meshing solution, but required more than twice the computation time. Based on these results, the reduced-z model (82,500 fine meshes) is used for all further analysis.

\subsection{Comparison of $\mathrm{S}_{\mathrm{N}}$ only with Hybrid Methods}

The $S_{N}$ only solution for the neutron flux spectrum averaged over the end of the duct is now compared with the hybrid $S_{N}$ and characteristics method and $S_{N}$ with fictitious quadrature set solutions. The spectrum calculated by each TITAN method is plotted along with the MCNP reference solution in Fig. 5.

In Fig. 5, the TITAN solutions match the shape of the MCNP solution well and, for groups 11-47 $(2.73-0 \mathrm{MeV})$, the TITAN solutions are within $17 \%$ of the MCNP solution. The TITAN result for the fictitious quadrature set is not accurate for low energy groups, approximately groups 30-47, because the method does not model the concrete around the duct. However, all three TITAN solutions show discrepancies in the first peak, i.e., groups 5-12 (2.37-8.61 MeV). To further examine this, the U-235 fission spectrum (source spectrum) is given along with the MCNP solution and the TITAN $\mathrm{S}_{\mathrm{N}}$ and characteristics method solution in Fig. 6.

The spectra predicted by TITAN closely follow the fission spectrum, but the MCNP solution for the first peak is different. This is believed to be due to the multigroup cross sections and the fact that the scattering moments cannot be directly used by MCNP, as discussed in Sect. 3.2.

\subsection{Computation Times}

The computation times for the different deterministic methods and speedup relative to the reference MCNP solution are given in Table 2. All calculations were done in parallel on 16 processors on a dedicated computer cluster. 


\section{$15^{\text {th }}$ ISRD}

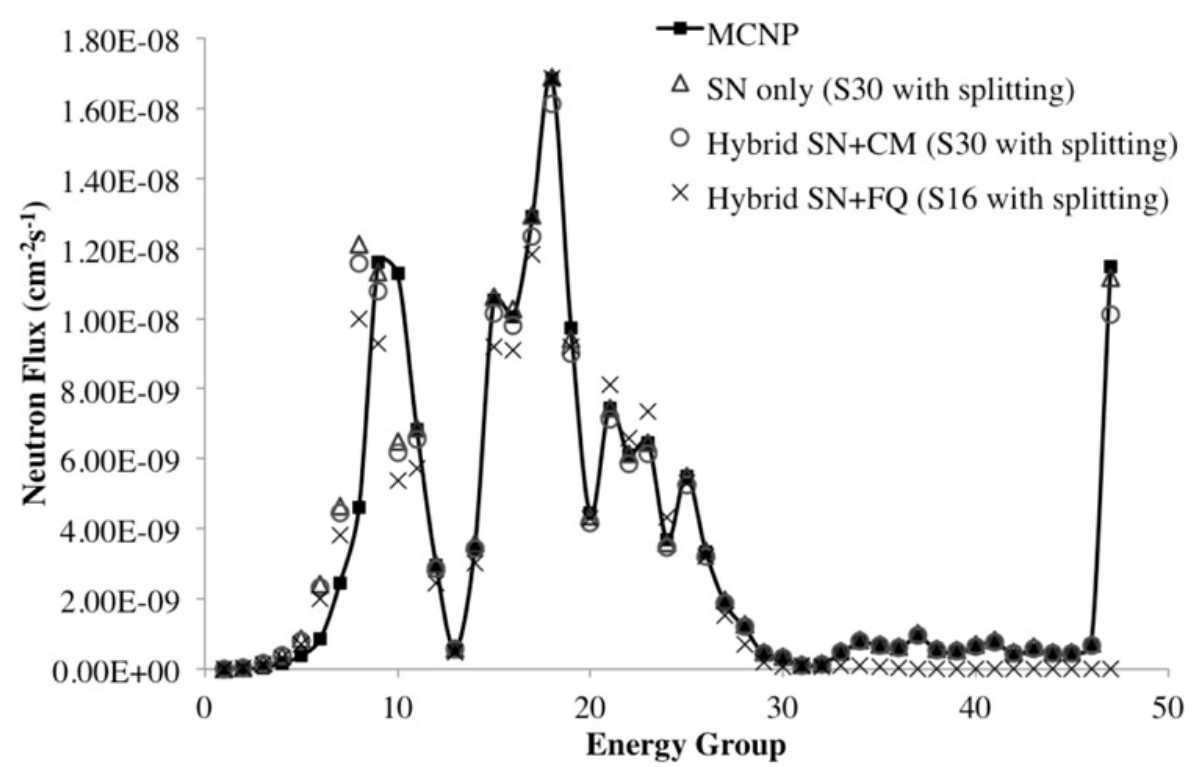

Figure 5. Flux spectra averaged over the end of the duct for MCNP, $S_{N}$ only, hybrid $S_{N}$ and characteristics method $(\mathrm{CM})$, and hybrid $\mathrm{S}_{\mathrm{N}}$ with fictitious quadrature $(\mathrm{FQ})$.

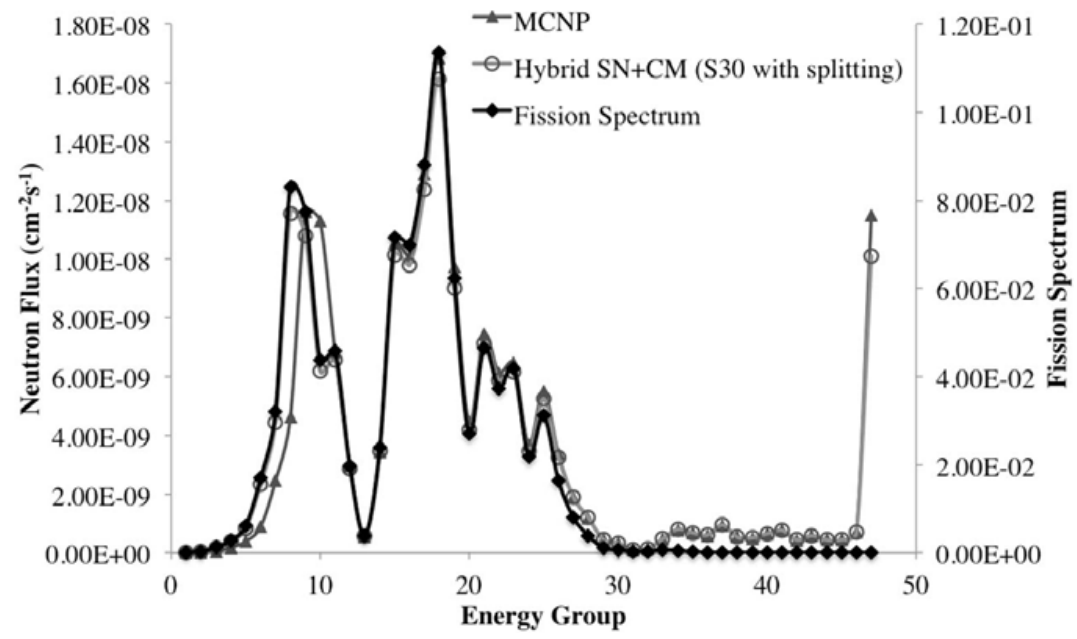

Figure 6. Flux spectra averaged over the end of the duct for MCNP and hybrid $\mathrm{S}_{\mathrm{N}}$ and characteristics method (CM) compared with the fission spectrum of the source.

Table 2. Computation times for different methods and speedup relative to MCNP on 16 processors.

\begin{tabular}{lcc}
\hline Solution Method & Computation Time (s) & Speedup \\
\hline MCNP5 & 64010 & - \\
TITAN $S_{N}$ & 1478 & 43.3 \\
TITAN $S_{N}+$ CM & 1319 & 48.5 \\
TITAN $S_{N}+$ FQ & 44 & 1471.5 \\
\hline
\end{tabular}




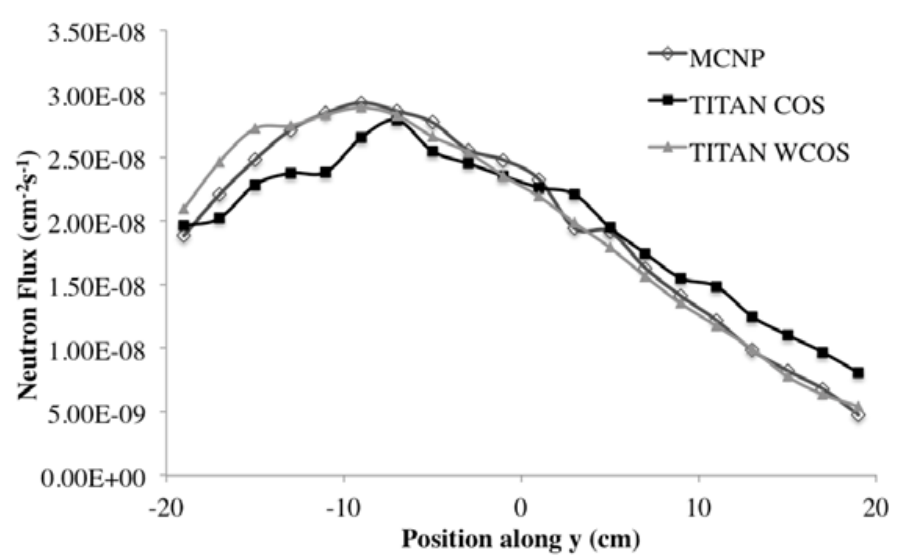

Figure 7. Flux distribution across end of duct for energy group $15(1.92-2.23 \mathrm{MeV})$ calculated by MCNP $(1 \sigma 2-5 \%)$, TITAN with COS, and TITAN with WCOS.

In Table 2, it is clear that all three TITAN methods show significant speedups relative to MCNP. The TITAN code with fictitious quadrature set was significantly faster than all methods presented here as a result of the reduced model size and number of directions.

\subsection{Collimated Detector Case}

To demonstrate the COS and WCOS techniques, a modified model with a non-uniform source and collimated detector at the end of the duct was created. The model geometry is identical to Fig. 2 except that the length is $198 \mathrm{~cm}$. The isotropic fission source spatially decreases along y and a collimator with an acceptance angle of $3.0^{\circ}$ restricts neutrons reaching the end of the duct.

The neutron flux across the end of the duct is given in Fig. 7 for MCNP, TITAN with COS, and TITAN with WCOS for energy group $15(1.92-2.23 \mathrm{MeV})$. The MCNP uncertainty is 2-5\% for this data. In general, the COS and WCOS techniques give similar results across energy groups, but the WCOS technique behaves more consistently, especially as the number of split directions is increased. Additional information on the WCOS technique can be found in reference 4.

\section{Conclusions}

The TITAN hybrid deterministic transport code's methods have been applied to streaming of neutrons through a duct in a nuclear power plant. The ordinate splitting technique has been shown to significantly reduce the number of directions needed to accurately model the duct and therefore reduce computation time. Three different methods of modelling the duct in TITAN have been demonstrated: $\mathrm{S}_{\mathrm{N}}$ only, $\mathrm{S}_{\mathrm{N}}$ and characteristics method, and $S_{N}$ with fictitious quadrature set. The calculated spectra at the end of the duct have been compared with a reference Monte Carlo solution. It has been shown that the TITAN solution methods have speedups of 43-1471 over the MCNP computation time, and the fastest computation is achieved by using $S_{N}$ with the fictitious quadrature set.

\section{References}

[1] C. Yi, A. Haghighat, Nucl. Sci. Eng. 164, 221 (2010)

[2] G. Longoni and A. Haghighat, Trans. Am. Nucl. Soc. 86, 246 (2002) 


\section{$15^{\text {th }}$ ISRD}

[3] C. Yi, A. Haghighat, $M \& C$ 2009, Saratoga Springs, NY, May 3-7 (2009)

[4] K. Royston, A. Haghighat, SNA+MC 2013, Paris, France, October 27-31 (2013)

[5] K. Royston, A. Haghighat, C. Yi, Prog. Nucl. Sci. Tech. 2, 201 (2011)

[6] F. Brown et al., Trans. Am. Nucl. Soc. 87, 273 (2002)

[7] C. Yi, XSMCNP, unpublished utility code (2009) 